\title{
ESQUARAÍNAS CIS E TRANS SUBSTITUÍDAS: APLICAÇÃO EXPERIMENTAL DA TEORIA DE GRUPO EM ESPECTROSCOPIA VIBRACIONAL
}

Vanessa E. de Oliveira ${ }^{a}$ e Luiz Fernando Cappa de Oliveira ${ }^{b, *}$,(1)

a'Departamento de Ciências da Natureza, Instituto de Humanidades e Saúde, Universidade Federal Fluminense, Campus Rio das Ostras, 28895-532 Rio das Ostras - RJ, Brasil

bDepartamento de Química, Instituto de Ciências Exatas, Universidade Federal de Juiz de Fora, 36036-900 Juiz de Fora - MG, Brasil

Recebido em 23/05/2019; aceito em 15/07/2019; publicado na web em 24/09/2019

\begin{abstract}
SQUARAINES CIS AND TRANS DERIVATIVES: PRACTICAL APPLICATION OF GROUP THEORY IN VIBRATIONAL SPECTROSCOPY. This work shows a practical application of the group theory to a relatively simple molecular system, as well its impact in the results obtained through vibrational spectroscopic techniques. Squaraines substituted by dicyanemethylene groups have been extensively studied, and after minor approaches in the sense to facilitate the point group determination, they were classified to the $\mathrm{C}_{2 \mathrm{v}}\left(\right.$ Cis) and $\mathrm{D}_{2 \mathrm{~h}}$ (Trans) groups. The Trans squaraine presents lower irreducible representations when compared to the Cis derivative; alterations in the arrangements of such systems promotes very interesting conjugation effects, since different reactivity from spectroscopic particularities. Especially to the Trans squaraine, there was a clear convergence between the data suggest by the calculations and those observed in their vibrational spectra, mainly that related to the inversion operation; strong bands visualized in a vibrational technique are not identified in other, mainly in region between $1800-1000 \mathrm{~cm}^{-1}$. The approach of local symmetry must to be adjust to better evaluation of the spectroscopic behavior of the Cis derivative, in this case, the theory suggests low symmetry, but in the spectra can be seen clearly a bigger symmetry. Then, the vibrational analysis by using different techniques was determinant to the discussion in a supramolecular level.
\end{abstract}

Keywords: squaraines; group theory; spectroscopy.

\section{OXOCARBONOS, PSEUDO-OXOCARBONOS E ESQUARAÍNAS}

Ácido esquárico $\left(\mathrm{H}_{2} \mathrm{C}_{4} \mathrm{O}_{4}\right)$ e seu íon divalente esquarato $\left(\mathrm{C}_{4} \mathrm{O}_{4}{ }^{2-}\right)$ é um dos mais importantes membros de uma família conhecida como "oxocarbonos", ${ }^{1-3}$ essa classe dianiônica é formada por outros três compostos todos contendo apenas átomos de carbono e oxigênio, Figura 1. A simplicidade e elegância estrutural destas espécies garante a esses sistemas moleculares uma elevada simetria e, por consequência, são ferramentas extremamente relevantes na abordagem espectroscópica.

Esses compostos são amplamente utilizados em diversos campos da ciência, desde o desenvolvimento de semicondutores a estudos fotoquímicos, como receptores de radiação;, ${ }^{2,4}$ sais de rodizonato são empregados como reagente analítico, por exemplo, na identificação de traços de chumbo em perícia criminal, em análises de rádio em águas doces, além de identificar ferro (II) e bário $;^{5}$ temos mais recentemente citações dos sais de rodizonato em baterias de alto desempenho. ${ }^{6,7}$ Utiliza-se o ácido esquárico como matéria-prima no desenvolvimento de materiais importantes em processos bioquímicos e fotocondutivos. ${ }^{8-13}$

Oxocarbonos são peculiares por apresentarem estruturas cíclicas e planas o que favorece a deslocalização dos elétrons em conjugação e possibilita a visualização de efeitos especiais, tais como o efeito Jahn-Teller. ${ }^{1-3,14-18}$ No caso das esquaraínas, o efeito ocorre devido à dupla degenerescência observada no estado eletrônico excitado de menor energia destas moléculas. Esse efeito é típico de sistemas altamente simétricos e pode ser facilmente verificado em seus espectros eletrônicos através do perfil da banda, um máximo com elevada absortividade, da ordem de $10^{4} \mathrm{~L} \mathrm{~mol}^{-1} \mathrm{~cm}^{-1}$, e um ombro na região de menor energia com absortividade molar também elevada. ${ }^{3,16,19}$ Essa dupla degenerescência também influencia alguns modos vibracionais e, portanto, pode ser verificada em seus espectros vibracionais mais especificamente através da técnica Raman. Em medidas realizadas fora da condição de ressonância percebe-se que modos não totalmente simétricos podem se apresentar com intensidade relativa equivalente

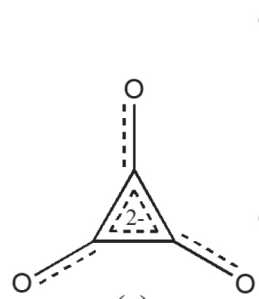

(a)

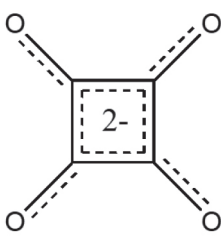

(b)<smiles>O=C1C(=O)C(=O)C(=O)C1=O</smiles>

(c)<smiles></smiles>

(d)

Figura 1. Representação dos diânions (a) deltato, (b) esquarato, (c) croconato e (d) rodizonato

*e-mail: luiz.oliveira@ufjf.edu.br 
ou até superior a modos simétricos. Um exemplo clássico é a banda atribuída ao estiramento $\mathrm{CO}$, um modo totalmente simétrico, mas ocorre como uma banda de fraca intensidade relativa quando comparados a modos não totalmente harmônicos, dentre eles, $v(C C)$ e $\delta(\mathrm{CC})_{\text {anel. }} \cdot 14,18,20$

Para abordar o tema, várias metodologias se mostraram valiosas para a determinação do grupo pontual destas espécies; assim, são frequentemente utilizados estudos vibracionais através das técnicas Raman e de absorção no infravermelho, cálculos de coordenadas normais e difração de raios X. ${ }^{18,21}$ Utilizando estes princípios, conseguiu-se, por exemplo, concluir que o íon esquarato possui simetria $\mathrm{D}_{4 \mathrm{~h}}$, o croconato $\mathrm{D}_{5 \mathrm{~h}}$, o rodizonato $\mathrm{D}_{6 \mathrm{~h}}$, o deltato $\mathrm{D}_{3 \mathrm{~h}} .{ }^{22-25}$

Dentro do contexto de simetria molecular, é quase imediata a verificação de semelhança no comportamento espectroscópico para os inúmeros derivados dos compostos em questão, principalmente para os íons esquarato e croconato. Várias destas substâncias foram sintetizadas desde a sua descoberta em meados do século XIX; ;6,27 alterações estruturais são mais facilmente encontradas para os anéis com 5 e 4 carbonos..$^{3,28-41}$ Os íons deltato e rodizonato são mais difíceis de trabalhar, o primeiro porque sua síntese é extremamente complicada; o segundo deve-se à excelente conjugação eletrônica conferida em seu anel de seis membros, formando compostos bastante estáveis. ${ }^{4,42-44}$

Pseudo-oxocarbonos é a classificação dada a quaisquer espécies derivadas dos membros originais $\left(\mathrm{C}_{\mathrm{n}} \mathrm{O}_{\mathrm{n}}\right)^{2-}$; toda alteração parcial ou total promovida na estrutura precursora causa severa influência em suas propriedades originais, principalmente do ponto de vista espectroscópico. ${ }^{2,3}$ Especificamente para o ácido esquárico, tem-se entre seus derivados uma subclasse chamada de "Esquaraínas"; são derivados nos quais ocorre a substituição de um ou mais átomos de oxigênio do diânion por grupos nitrogenados, independente do restante da estrutura do grupo substituinte..$^{3,35,45,46} \mathrm{O}$ ácido esquárico por si só possui uma estrutura muito interessante; a modificação dessa estrutura primitiva pode trazer novas propriedades físico-químicas, bem como a intensificação de outras características pré-existentes.

Alguns exemplos podem ser encontrados na Figura 2; nesse caso estão apresentadas cinco variações de estruturas passíveis de serem obtidas a partir do reagente malononitrila $\left(\mathrm{CH}_{2}(\mathrm{CN})_{2}\right)$, o grupamento dicianometileno (DCM) é inserido no anel esquarato através do átomo de carbono não nitrogenado.
A introdução de grupos contendo elétrons $\pi$ aumenta a extensão da forte deslocalização eletrônica pré-existente em um sistema do tipo doador-receptor-doador $(D-R-D)$ de elétrons, gerando compostos intensamente coloridos e com elevada fluorescência. ${ }^{45,47-50}$ As esquaraínas pertencem a uma classe de compostos excelentes fotocondutores orgânicos; cálculos semi-empíricos de orbitais moleculares mostram que ambos os seus estados fundamental e excitado são estados com transferência de carga intramolecular do tipo doador-receptor-doador, $D-R-D^{50}$. Esse tipo de composto absorve intensamente próximo a $600 \mathrm{~nm}$ apresentando valores de absortividade molar, $\varepsilon$, da ordem de $10^{5}$, além de intensa fluorescência e mínimos deslocamentos Stokes. ${ }^{50}$

Dentro de nosso grupo de estudo, podem ser destacados alguns trabalhos que abordam a síntese deste tipo de esquaraínas; $;^{3,34-36,51,52}$ a grande maioria dos trabalhos disponíveis na literatura envolvem derivados do tipo Trans di-substituídas, em que o anel central encontra-se cercado por dois grupos substituintes doadores de elétrons na conformação $D-R-D$. Esses tipos de esquaraínas apresentam absorções na região do vermelho do espectro eletromagnético com valores de $\varepsilon>200.000 \mathrm{~L} \mathrm{~mol}^{-1} \mathrm{~cm}^{-1}$, além de serem consideravelmente fotoestáveis $;^{53}$ tais especificidades os habilitam como fortes candidatos na aplicação em xerografia ou na confecção de lasers de diodo.

O objetivo do presente estudo é tecer uma comparação entre os dados obtidos na aplicação do estudo de teoria de grupo e os dados empíricos obtidos nas medidas de espectroscopia Raman, fora da ressonância eletrônica. Para tanto foram consideradas duas esquaraínas Cis e Trans di-substituídas por grupos dicianometileno (DCM); ambos os compostos foram extensivamente investigados e caracterizados através de diversificadas técnicas em trabalhos anteriores. ${ }^{3,35,36}$

\section{ESPECTROSCOPIA MOLECULAR E TEORIA DE GRUPO}

Dentre as técnicas mais utilizadas para investigação das características vibracionais das moléculas estão as espectroscopias de absorção no infravermelho e a Raman (ou espalhamento Raman). Na espectroscopia vibracional obtêm-se informações sobre as ligações químicas através dos valores das frequências das vibrações ou ainda das constantes de força do oscilador responsável pela transição, além de informações relacionadas à geometria molecular investigando o número de modos vibracionais ativos no infravermelho e Raman..$^{54-59}$ Ambas são técnicas permitem a identificação das mais variadas

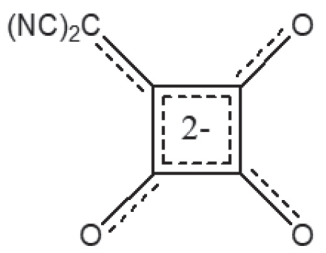

(a)<smiles></smiles>

(b)

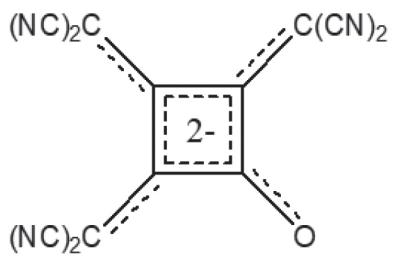

(d)<smiles>CC(C)=C1C(=CN)C(=CCN)C1=C(C)C</smiles>

(e)

Figura 2. Estruturas moleculares dos diânion das esquaraínas: (a) mono(DCM) esquarato; (b) Trans bis(DCM) esquarato; (c) cis bis(DCM) esquarato; (d) tris(DCM) esquarato e (e) tetra(DCM) esquarato 
substâncias simplesmente através do seu espectro vibracional; são consideradas técnicas complementares uma vez que os princípios físicos para a obtenção destas informações vibracionais são diferentes.

\section{INSTRUMENTAÇÃO E SÍNTESE}

Espectroscopia Vibracional no Infravermelho: Os compostos sintetizados foram submetidos à análise de absorção na região do infravermelho em um espectrômetro Bomen FTIR MB 102, na região de 4000 - $400 \mathrm{~cm}^{-1}$ com número médio de varreduras de 128 e resolução espectral de $4 \mathrm{~cm}^{-1}$ preparados em pastilhas com $\mathrm{KBr}$.

Espectroscopia Raman: As medidas foram realizadas em linha de excitação $1064 \mathrm{~nm}$, usando como equipamento um espectrômetro Bruker RFS 100 com transformada de Fourier, laser Nd:YAG operando em $1064 \mathrm{~nm}$ com potência nominal de até $1000 \mathrm{~mW}$ e detector de Germânio; média de 1000 espectros com resolução espectral de $4 \mathrm{~cm}^{-1}$.

Espectroscopia Eletrônica: Os espectros eletrônicos foram obtidos em um espectrofotômetro Shimadzu UVPC 1601, com lâmpada de halogênio (infravermelho próximo e visível) e de deutério (ultravioleta), com detectores do tipo fotomultiplicadora (ultravioleta e visível) e cela de PbS (infravermelho próximo). Para tomada dos espectros foram utilizadas cubetas de quartzo com caminho óptico de $10 \mathrm{~mm}$, e resolução de $2 \mathrm{~nm}$.

Ressonância Magnética Nuclear de ${ }^{13} \mathrm{C}$ de ${ }^{1} \mathrm{H}$ : Os espectros de ressonância magnética nuclear de ${ }^{1} \mathrm{H}$ e de ${ }^{13} \mathrm{C}$ foram registrados em equipamento Bruker AC-300, operando nas frequências de 300,13 e 75,43 MHz, respectivamente, pertencente ao Departamento de Química da Universidade Federal de Juiz de Fora.

Análise elementar: As análises dos teores percentuais de C, $\mathrm{He} \mathrm{N}$ foram realizadas em aparelho Perkin Elmer modelo 2400 na Central analítica do Instituto de Química da Universidade de São Paulo.

Análises termogravimétricas: Análises preliminares de ponto de fusão foram realizadas em um aparelho digital MICROQUÍMICA, modelo MQAPF-301, pertencente ao Departamento de Química da Universidade Federal de Juiz de Fora. As análises termogravimétricas foram realizadas em equipamento TG-50 Mettler STARe. As amostras foram aquecidas a partir da temperatura ambiente até $800{ }^{\circ} \mathrm{C}$, em uma faixa de $10{ }^{\circ} \mathrm{C} \mathrm{min}{ }^{-1}$ em atmosfera de fluxo dinâmico de ar, em colaboração com o Laboratório de Análises Térmicas coordenado pela Professora Maria Irene Yoshida do Departamento de Química do Instituto de Ciências Exatas da Universidade Federal de Minas Gerais.

Sintese das esquaraínas:

Trans: Duas etapas: Etapa (1) Síntese do intermediário trans dianilino esquarato de sódio realizado de acordo com a literatura. ${ }^{60}$ Etapa (2) Em um balão de fundo redondo adicionar $200 \mathrm{mg}$ de dianilino esquarato de sódio e $75 \mathrm{~mL}$ de etanol anidro, aquecer até a temperatura de ebulição; em um béquer solubilizar 100 mg de malononitrila em $15 \mathrm{~mL}$ de etanol tratado; em outro béquer adicionar $10 \mathrm{~mL}$ de etanol a $50 \mathrm{mg}$ de sódio metálico (excesso), após cessar a efervescência adicionar simultaneamente estas duas soluções ao balão reacional contendo o intermediário dianilino esquarato; Deixar sob refluxo por 1,5 h. Após este período, interromper o processo em banho de gelo. O sólido alaranjado obtido deverá ser solubilizado em etanol (150 mL); após filtragem, recolher a água de lavagem e colocar em geladeira por 5 h. A esquaraína Trans cristalizará nesta solução; proceder com filtragens destas recristalizações. Cor: alaranjado. Rendimento: $53 \%$. $\mathrm{Na}_{2} \mathrm{C}_{10} \mathrm{~N}_{4} \mathrm{O}_{2} \cdot 4 \mathrm{H}_{2} \mathrm{O}$ » $\mathbf{C H N / e n t r e ~ p a r e ̂ n t e s e s ~ o s ~}$ valores teóricos: C: 37,13 (36,82); H: 2,45 $(2,47)$ e N: 15,90 $(17,18)$. TG » Perdas de massa: 35 e $85^{\circ} \mathrm{C}\left[4 \mathrm{H}_{2} \mathrm{O}\right]=21,76 \%$ (21,49\%); última perda de massa em $500{ }^{\circ} \mathrm{C}$ [Resíduo $\mathrm{Na}_{2} \mathrm{CO}_{3}$ ] $=31,08 \%(32,51 \%)$. UV-vis (Água): $\lambda_{1}=424 \mathrm{~nm}\left(\varepsilon=2,4.10^{5} \mathrm{~mol}^{-1} \mathrm{~L} \mathrm{~cm}^{-1}\right) ; \lambda_{2}=304 \mathrm{~nm}$ $\left(\varepsilon=5,0 \times 10^{4} \mathrm{~mol}^{-1} \mathrm{~L} \mathrm{~cm}^{-1}\right) ; \lambda_{3}=228 \mathrm{~nm}\left(\varepsilon=1,6.10^{4} \mathrm{~mol}^{-1} \mathrm{~L} \mathrm{~cm}^{-1}\right)$. $\mathbf{R M N}{ }^{13} \mathbf{C}\left(75,43 \mathrm{MHz}, \mathrm{D}_{2} \mathrm{O}\right): \delta(\mathrm{ppm})=172,6 ; 166,0 ; 121,0 ; 35.3$.

Cis: Duas etapas: Etapa (1) Síntese do intermediário dibutilesquarato: Em um balão de fundo redondo adicionar 1,00 g de ácido esquárico e $70 \mathrm{~mL}$ de butanol bidestilado, manter sob refluxo em aparelhagem contendo uma trap (Dean Stark) por 24 h; após este período resfriar o balão em banho de gelo. Etapa (2) Em um béquer solubilizar $630 \mathrm{mg}$ de malononitrila em $20 \mathrm{~mL}$ de butanol bidestilado; em outro béquer adicionar $10 \mathrm{~mL}$ de butanol a $50 \mathrm{mg}$ de sódio metálico (excesso), após cessar a efervescência adicionar simultaneamente estas duas soluções ao balão reacional contendo o intermediário dibutilesquarato previamente resfriado. Deixar sob agitação por 10 minutos em temperatura ambiente; filtrar e lavar com butanol. Rendimento: 93\%. $\mathrm{Na}_{2} \mathrm{C}_{10} \mathrm{~N}_{4} \mathrm{O}_{2} \cdot 3 \mathrm{H}_{2} \mathrm{O}$ » $\mathbf{C H N} /$ entre parênteses os valores teóricos: C: $38,39(38,98)$; H: 1,91 (1,96) e N: 17,47 $(18,18)$. TG » Perdas de massa: $30-95{ }^{\circ} \mathrm{C}\left[3 \mathrm{H}_{2} \mathrm{O}\right]=20,0 \%(17,5 \%)$; última perda $>500{ }^{\circ} \mathrm{C}$ [resíduo $\mathrm{Na}_{2} \mathrm{CO}_{3}$ ] $=36,7 \%(34,4 \%)$. UV-vis (Água): $\lambda_{1}=382 \mathrm{~nm}(\varepsilon$ $\left.=4,2 \times 10^{4} \mathrm{~mol}^{-1} \mathrm{~L} \mathrm{~cm}^{-1}\right) ; \lambda_{2}=347 \mathrm{~nm}\left(\varepsilon=3,1 \times 10^{4} \mathrm{~mol}^{-1} \mathrm{~L} \mathrm{~cm}^{-1}\right)$; $\lambda_{3}=263 \mathrm{~nm}\left(\varepsilon=2,0 \times 10^{4} \mathrm{~mol}^{-1} \mathrm{~L} \mathrm{~cm}^{-1}\right) ; \lambda_{4}=228 \mathrm{~nm}\left(\varepsilon=2,6.10^{4} \mathrm{~mol}^{-1}\right.$ $\left.\mathrm{L} \mathrm{cm}^{-1}\right)$. RMN ${ }^{13} \mathbf{C}\left(75,43 \mathrm{MHz}, \mathrm{D}_{2} \mathrm{O}\right): \delta(\mathrm{ppm})=187,0 ; 173,0 ; 124,0$; 39,2 (Material Suplementar, Figuras S1 e S2).

\section{DISCUSSÕES}

O tipo de simetria molecular que os íons apresentam é determinante para uma melhor discussão sobre os espectros vibracionais, uma vez que variações na simetria provocam mudanças no número bandas observadas nos espectros. ${ }^{56,57,61-64}$ Efetuando os cálculos das representações vibracionais para os ligantes e, considerando-os como diânions livres (Figura 3), encontra-se 42 modos vibracionais (3N-6):

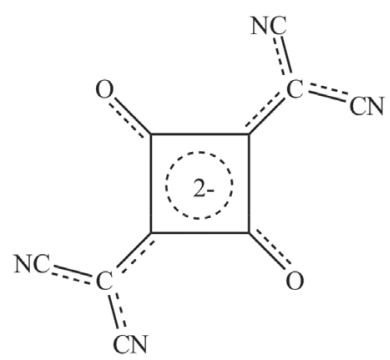

(a)

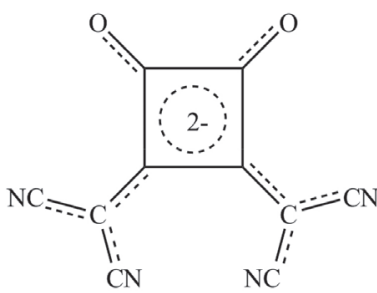

(b)
Figura 3. Íons (bis)dicianometileno esquarato (a) Trans e (b) Cis

Representações vibracionais (diânion livre):

Trans - simetria $\mathrm{D}_{2 \mathrm{~h}}$

$\Gamma=8 \mathrm{Ag}+7 \mathrm{~B} 1 \mathrm{~g}+3 \mathrm{~B} 2 \mathrm{~g}+3 \mathrm{~B} 3 \mathrm{~g}+7 \mathrm{~B} 2 \mathrm{u}+2 \mathrm{Au}+7 \mathrm{~B} 3 \mathrm{u}+5 \mathrm{~B} 1 \mathrm{u}$

$\Gamma_{\mathrm{IV}}=2 \mathrm{Au}+7 \mathrm{~B} 2 \mathrm{u}+7 \mathrm{~B} 3 \mathrm{u}+5 \mathrm{~B} 1 \mathrm{u} 21$ representações.

$\Gamma_{\text {Raman }}=8 \mathrm{Ag}+7 \mathrm{~B} 1 \mathrm{~g}+3 \mathrm{~B} 2 \mathrm{~g}+3 \mathrm{~B} 3 \mathrm{~g} 21$ representações. »» $\mathrm{IV}_{21} \mathrm{e}$ $\operatorname{Raman}_{21}$

Cis - simetria $\mathrm{C}_{2 \mathrm{v}}$

$\Gamma=15 \mathrm{~A}_{1}+7 \mathrm{~A}_{2}+6 \mathrm{~B}_{1}+14 \mathrm{~B}_{2}$

$\Gamma_{\mathrm{IV}}=15 \mathrm{~A}_{1}+14 \mathrm{~B}_{2}+6 \mathrm{~B}_{1} 35$ representações.

$\Gamma_{\text {Raman }}=15 \mathrm{~A}_{1}+7 \mathrm{~A}_{2}+14 \mathrm{~B}_{2}+6 \mathrm{~B}_{1} \Rightarrow 42$ representações. "» $\mathrm{IV}_{42} \mathrm{e}$ $\operatorname{Raman}_{35}$

Assim, tem-se o Cis pertencente ao grupo de ponto $\mathrm{C}_{2 v}$ e o Trans ao grupo $\mathrm{D}_{2 \mathrm{~h}}$; esperava-se que experimentalmente houvesse um número maior de bandas nos espectros do composto Cis em relação ao composto Trans. A aproximação mencionada e os resultados oriundos da abordagem adotada no cálculo sugerem que peculiaridades 
espectroscópicas são esperadas para ambos os ligantes. Por exemplo, para o ligante $C i s$ espera-se que haja uma diferença significativa no número de bandas verificadas em seus espectros vibracionais. Além disso, a presença do elemento de simetria inversão verificado no cálculo para o ligante Trans vem sugerir que, experimentalmente, os espectros apresentem bandas ativas em uma técnica vibracional e inativas em outra. Nas Figuras 4, 5 e 6 estão apresentados os espectros vibracionais experimentais para ambos os ligantes (contra íon sódio).

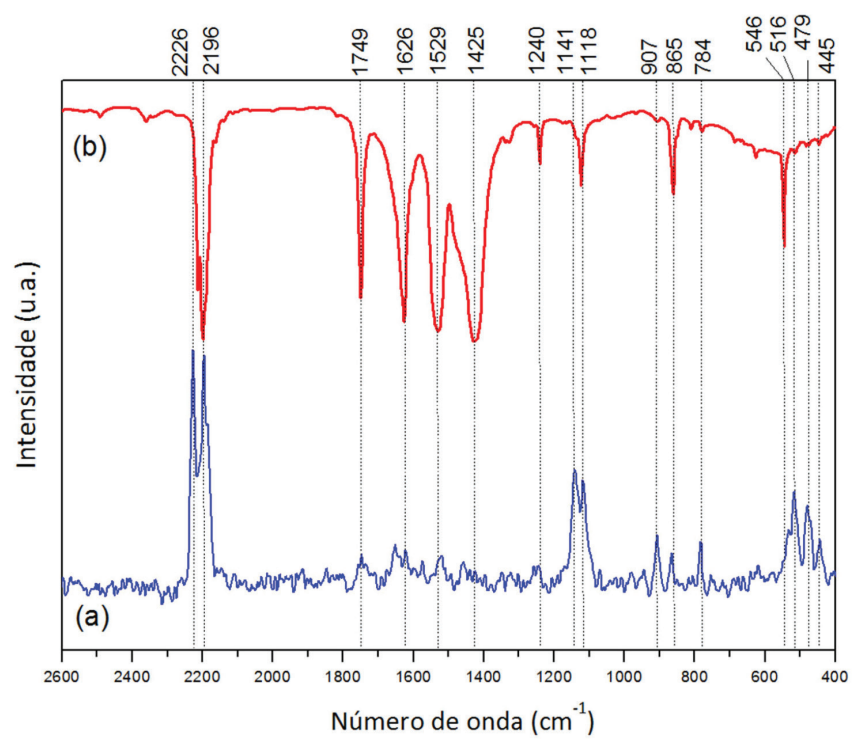

Figura 4. Espectros (a) Raman e (b) de absorção no infravermelho para o Cis-Na, linha de excitação $1064 \mathrm{~nm}$
Na Tabela 1 estão sumarizados os valores de frequência Raman e IV para os ligantes Trans e Cis, bem como a atribuição de bandas com suas respectivas intensidades relativas.

A análise conjunta dos espectros experimentais endossou a proposta teórica de elevada simetria. Salienta-se a presença de bandas de elevada intensidade relativa no espectro de IV e inativas no espectro Raman (região entre 1750 e $1420 \mathrm{~cm}^{-1}$ ). No caso do composto Trans constata-se a existência do elemento de simetria "centro de

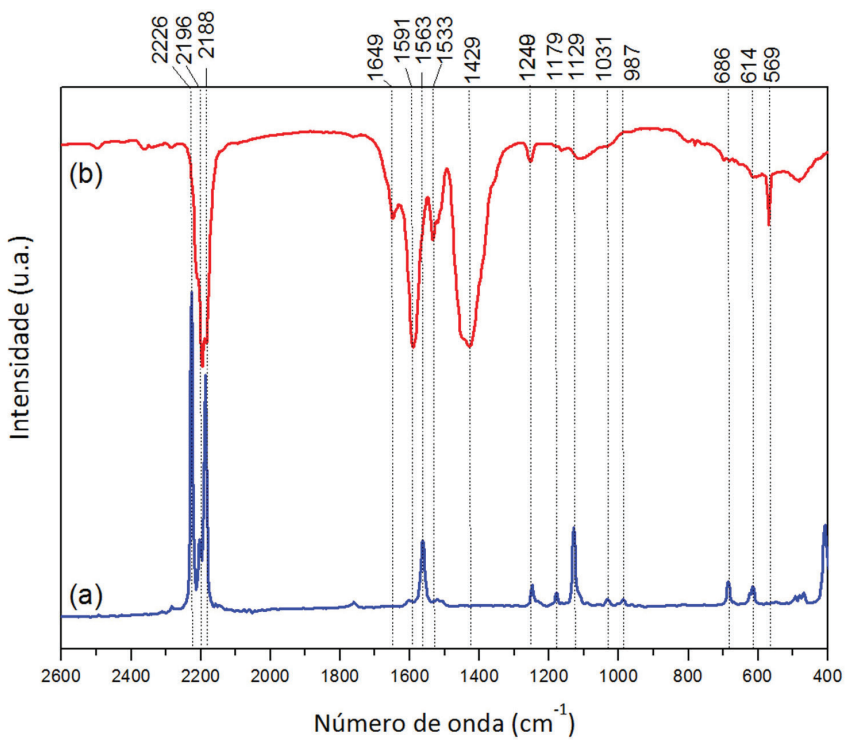

Figura 5. Espectros (a) Raman e (b) de absorção no infravermelho para o Trans-Na, linha de excitação $1064 \mathrm{~nm}$

Tabela 1. Valores de números de onda $\left(\mathrm{em} \mathrm{cm}^{-1}\right)$ Raman e IV para os ligantes Trans e Cis, e atribuição de bandas com suas respectivas intensidades relativas*

\begin{tabular}{|c|c|c|c|c|}
\hline \multicolumn{2}{|c|}{ IV } & \multicolumn{2}{|c|}{ Raman } & \multirow[t]{2}{*}{ Atribuição } \\
\hline Trans & Cis & Trans & Cis & \\
\hline $482 \mathrm{w}$ & $446 \mathrm{w} / 472 \mathrm{w} / 482 \mathrm{w}$ & $469 \mathrm{w} / 482 \mathrm{w}$ & 443 w/494 w & $\delta$ do anel no plano \\
\hline- & $513 \mathrm{w}$ & - & $516 \mathrm{~m}$ & \\
\hline $568 \mathrm{~m}$ & $546 \mathrm{~m}$ & - & $531 \mathrm{sh}$ & \\
\hline 609 w/683 w/699w & 626 w/688 w & 614 w/686 w & - & Respiração do anel \\
\hline $781 \mathrm{w}$ & $779 \mathrm{w}$ & - & $784 \mathrm{~m}$ & \\
\hline \multirow[t]{2}{*}{-} & $810 \mathrm{w} / 860 \mathrm{~m}$ & - & $865 \mathrm{w}$ & \\
\hline & $908 \mathrm{w}$ & - & $907 \mathrm{w}$ & \\
\hline $1109 w$ & $1122 \mathrm{~m}$ & $1034 \mathrm{w} / 1129 \mathrm{~m}$ & - & \\
\hline $1164 \mathrm{w}$ & - & $1178 \mathrm{w}$ & $1141 \mathrm{~m}$ & $v(\mathrm{CC})$ \\
\hline $1252 \mathrm{w}$ & $1241 \mathrm{w}$ & $1249 w$ & - & \\
\hline $1358 \mathrm{sh}$ & $1338 \mathrm{w} / 1327 \mathrm{w}$ & - & - & \\
\hline $1386 \mathrm{sh}$ & - & - & - & \\
\hline $1427 \mathrm{~s}$ & $1427 \mathrm{~s}$ & - & - & $v[(\mathrm{CC})+(\mathrm{CO})+(\mathrm{CC}(\mathrm{CN}))]$ \\
\hline $1449 \mathrm{sh} / 1510 \mathrm{sh}$ & $1469 \mathrm{sh}$ & - & - & $v[(\mathrm{CC})+(\mathrm{CO})+(\mathrm{CC}(\mathrm{CN}))]$ \\
\hline $1519 \mathrm{sh}$ & - & $1521 \mathrm{w}$ & - & $v[(\mathrm{CC})+(\mathrm{CO})+(\mathrm{CC}(\mathrm{CN}))]$ \\
\hline $1533 \mathrm{~m}$ & $1528 \mathrm{~s}$ & - & - & $v[(\mathrm{CC})+(\mathrm{CO})+(\mathrm{CC}(\mathrm{CN}))]$ \\
\hline $1591 \mathrm{~s}$ & - & $1563 \mathrm{~m}$ & - & $v[(\mathrm{CC})+(\mathrm{CO})+(\mathrm{CC}(\mathrm{CN}))]$ \\
\hline $1650 \mathrm{w}$ & $1626 \mathrm{~s}$ & - & $1652 \mathrm{w}$ & $v[(\mathrm{CC})+(\mathrm{CO})+(\mathrm{CC}(\mathrm{CN}))]$ \\
\hline- & $1750 \mathrm{~s}$ & - & $1748 w$ & $v(\mathrm{CO})$ \\
\hline $2184 \mathrm{sh} / 2195 \mathrm{~s}$ & $2163 \mathrm{sh} / 2199 \mathrm{~s}$ & $2188 \mathrm{~s} / 2204 \mathrm{~s}$ & $2185 \mathrm{sh} / 2196 \mathrm{~s}$ & $v(\mathrm{CN})$ \\
\hline $2211 \mathrm{sh}$ & $2213 \mathrm{~s}$ & $2226 s$ & $2226 \mathrm{~s}$ & $v(\mathrm{CN})$ \\
\hline
\end{tabular}

*s: forte, m: média, w: fraca e sh: ombro. 
inversão", como predito pelos cálculos supracitados; demonstrando assim, que a aproximação feita é válida para predizer informações espectroscópicas.

Para o ligante Cis observa-se que a região entre 1800 a $1300 \mathrm{~cm}^{-1}$ do espectro de absorção no infravermelho é mais rica em bandas (Figura 4b), e essas são significativamente mais intensas e definidas que as bandas observadas em seu espectro Raman, Figura 4a. Essa constatação pode indicar uma assimetria local em consequência da menor deslocalização eletrônica no anel oxocarbônico sugerida para o ligante Cis. Isto porque, além da questão da intensidade já comentada, há um aumento no número destas bandas associadas aos modos de vibração atribuídos especificamente aos átomos do anel desta esquaraína. Na região entre 1750 e $1427 \mathrm{~cm}^{-1}$ observa-se a existência de bandas ativas no IV e inativas ou pouco permitidas no espectro Raman. Na região de menores números de onda também são observadas bandas com esta característica. $\mathrm{Na}$ aproximação feita para os cálculos teóricos utilizando teoria de grupo, a análise do grupo para o diânion livre $C i s$ (grupo $\mathrm{C}_{2 \mathrm{v}}$ ) não prevê o elemento de simetria centro de inversão; porém, a análise em conjunto dos espectros vibracionais sugere a sua presença. O centro de inversão não verificado na aproximação para simetria molecular local poderia ser confirmado por análise de difração de raios X. O resultado demonstraria a presença deste elemento de simetria na célula unitária envolvendo mais de uma molécula; porém, a obtenção de monocristais da esquaraína com sódio não foi bem-sucedida.

Para ambos os ligantes, destacam-se pelo menos duas bandas intensas entre 2230 e $2180 \mathrm{~cm}^{-1}$ atribuídas ao modo estiramento CN; a modificação na posição dos grupos dicianometileno substituintes em Trans ou Cis implica em poucas alterações no valor do número de onda do modo de estiramento destas nitrilas, como pode ser observado na ampliação da região entre $2300-2100 \mathrm{~cm}^{-1}$ na Figura 6 . Observam-se valores muito próximos para essas vibrações, independente da posição do grupo substituinte e, de modo geral, podem ser observadas bandas melhor definidas e relativamente mais intensas nos respectivos espectros Raman. Pode-se salientar a banda definida e relativamente intensa em $2188 \mathrm{~cm}^{-1}$ no espectro Raman para a esquaraína Trans, banda esta que se apresenta como um ombro no espectro de absorção IV. Dados analíticos de análise elementar de $\mathrm{CHN}$ e termogravimétrica sugerem a presença de moléculas de água de hidratação na estrutura das duas esquaraínas: $\mathrm{Na}_{2} \mathrm{C}_{10} \mathrm{~N}_{4} \mathrm{O}_{2} \cdot 4 \mathrm{H}_{2} \mathrm{O}$ e $\mathrm{Na}_{2} \mathrm{C}_{10} \mathrm{~N}_{4} \mathrm{O}_{2} \cdot 3 \mathrm{H}_{2} \mathrm{O} \cdot{ }^{35,36}$
Bandas na região entre 1650 e $1400 \mathrm{~cm}^{-1}$ são atribuídas aos modos acoplados $\mathrm{C}=\mathrm{C}, \mathrm{C}=\mathrm{O}$ e $\mathrm{CC}(\mathrm{CN})_{2}$; observa-se que não há variação significativa nos valores dos números de onda dos estiramentos $\mathrm{CC}$ e $\mathrm{CO}$, em função das diferentes substituições. Comparativamente, a esquaraína Trans apresenta um número menor de bandas em seus espectros, sugerindo maior efeito de conjugação eletrônica na estrutura do pseudo-oxocarbono. Há uma interessante variação na banda referente ao modo estiramento da carbonila. Para a esquaraína Trans, tem-se uma banda fraca em $1650 \mathrm{~cm}^{-1}$ (além do $\mathrm{v}(\mathrm{CO})$ nos demais modos acoplados), e na Cis essa banda se encontra em $1750 \mathrm{~cm}^{-1}$ e é relativamente muito intensa, sugerindo um caráter de dupla ligação. A fraca intensidade relativa desta banda próximo a $1750 \mathrm{~cm}^{-1}$ nos espectros do composto Trans sugere uma contribuição maior para os modos que ocorrem acoplados: $v[(\mathrm{CC})+(\mathrm{CO})+(\mathrm{CC}(\mathrm{CN}))]$. $\mathrm{O}$ abaixamento da simetria molecular para o derivado $\mathrm{Cis}$ justificaria a verificação da referida banda para o modo puro de $v(\mathrm{CO})$ nos espectros vibracionais. Destaca-se que a carbonila apresenta uma banda com elevada polaridade e, portanto, pouco polarizável, justificando sua ausência no espectro Raman da esquaraína Cis substituída. De modo geral, o alargamento de algumas bandas verificado nos espectros vibracionais é comum em compostos hidratados e ocorre devido aos possíveis acoplamentos existentes entre as esquaraínas e as moléculas de águas.

Lunelli e colaboradores ${ }^{45}$ realizaram um estudo vibracional ab initio para o diânion Trans; os autores obtiveram a estrutura cristalina do composto e observaram que o mesmo se apresenta em um plano e as carbonilas encontram-se ao longo do eixo de simetria de ordem 2; efetuando algumas aproximações chega-se a uma simetria $D_{2 h}$ para o respectivo diânion. $\mathrm{O}$ anel apresentou ligações equivalentes e com significativo caráter de dupla ligação, 1,457 ̊, o mesmo foi constatado para a distância $\mathrm{C}$ (anel)- $\mathrm{C}(\mathrm{CN})$. Apesar de alguns desvios da idealidade apontados no estudo, considera-se a aproximação muito boa e os desvios são correlacionados às diferentes graduações de carga molecular que, no caso da esquaraína Trans, estão centradas nos átomos de oxigênio e nitrogênio, Figura 7.

Para o derivado Cis, esse estudo cita que a distribuição de carga elétrica pelas ligações da molécula é nitidamente diferenciada. As médias de distâncias carbono/carbono do anel e as distâncias destes aos grupos $\mathrm{C}(\mathrm{CN})_{2}$ são de $1,422 \AA$, portanto, relativamente menores que os valores encontrados para a esquaraína Trans. A distância de separação CO - CO (entre as duas carbonilas) no Cis é maior que no
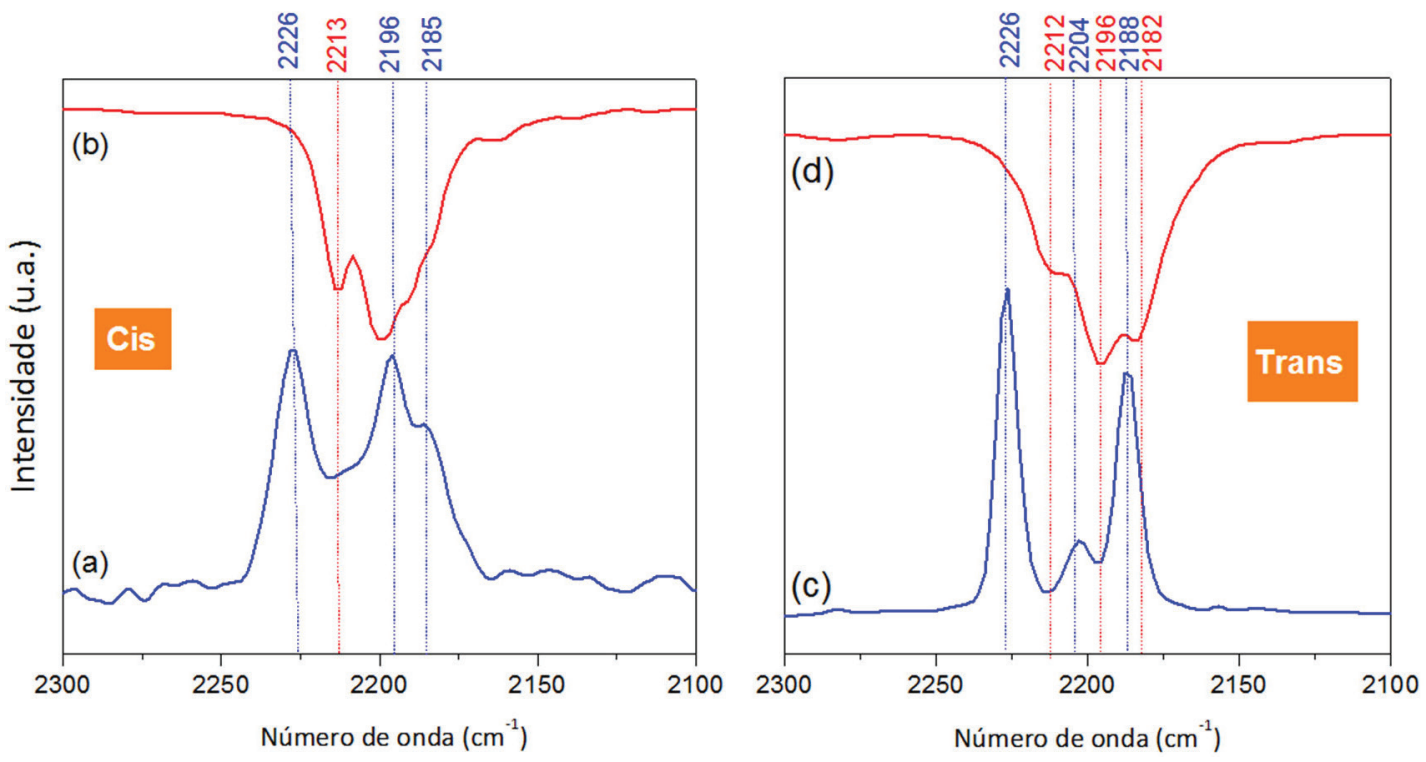

Figura 6. Espectros $(a) /(c)$ Raman e $(b) /(d)$ de absorção no infravermelho para os ligantes Cis e Trans, respectivamente 


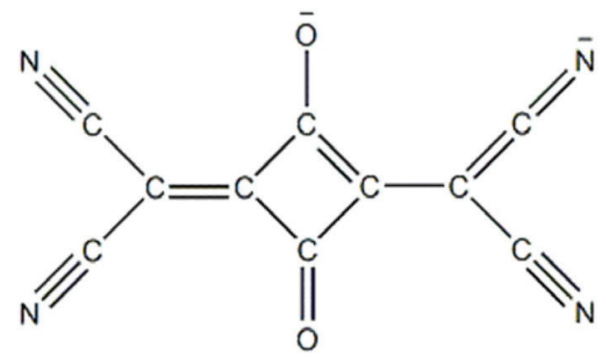

Figura 7. Forma canônica representando a concentração de carga negativa para o íon Trans

Trans (igual a 1,475 ̊̊). Por outro lado, a distância de ligação entre o átomo de carbono do anel e o átomo de carbono do grupo $-\mathrm{C}(\mathrm{CN})_{2}$ demonstra um caráter de dupla ligação significativo.

Os espectros eletrônicos para os sais de sódio das esquaraínas Cis e Trans, obtidos em solução aquosa, estão representados na Figura 8. Para o derivado Trans são observadas três transições bem distintas, sendo a primeira com elevada absortividade molar ocorrendo em $424 \mathrm{~nm}\left(\varepsilon=2,4.10^{5} \mathrm{~mol}^{-1} \mathrm{~L} \mathrm{~cm}^{-1}\right)$, as demais ocorrem em 304 e em 228 nm. O espectro eletrônico da Cis apresenta três transições bem distintas: o máximo de absorção é observado em $382 \mathrm{~nm}\left(\varepsilon=4,2 \times 10^{4} \mathrm{~mol}^{-1} \mathrm{~L} \mathrm{~cm}^{-1}\right)$ com um ombro em $347 \mathrm{~nm}\left(\varepsilon=3,1 \times 10^{4} \mathrm{~mol}^{-1} \mathrm{~L} \mathrm{~cm}^{-1}\right)$, a segunda e terceiras absorções ocorrem em $263 \mathrm{~nm}\left(\varepsilon=2,0 \times 10^{4} \mathrm{~mol}^{-1} \mathrm{~L} \mathrm{~cm}^{-1}\right)$ e $228 \mathrm{~nm}$ $\left(\varepsilon=2,6 \times 10^{4} \mathrm{~mol}^{-1} \mathrm{~L} \mathrm{~cm}^{-1}\right)$, respectivamente.

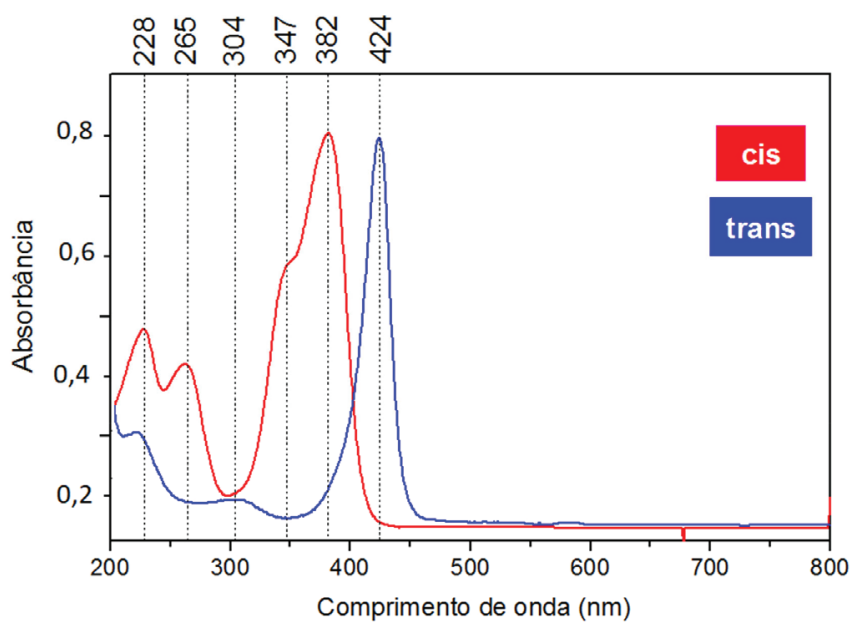

Figura 8. Espectro eletrônico para as esquaraínas indicadas

Como mencionado anteriormente, uma peculiaridade dos espectros eletrônicos dos íons oxocarbonos é a apresentação de um máximo de absorção associado com um ombro em regiões de maior energia. Esse perfil espectroscópico ocorre devido ao acoplamento vibrônico em estados eletrônicos excitados degenerados que é uma característica do espectro eletrônico destes compostos. . $^{1-3,14,16-20,35,65-68}$ No caso das duas esquaraínas em questão é esperada uma diminuição da simetria molecular devido à introdução de novos grupos, as esquaraínas mantém a excelente absortividade molar característica do precursor. Salienta-se a presença de um ombro com elevada absortividade molar (da ordem de $10^{4}$ ) tornando difícil sugerir um único grupo cromofórico na molécula responsável por esta transição eletrônica. Pode-se sugerir não se tratar do efeito Jahn-Teller, uma vez que esse derivado apresenta simetria ainda menor que o Trans e, este por sua vez de simetria mais baixa que a do íon esquarato.

A comparação dos espectros eletrônicos das esquaraínas sintetizadas permite a observação de um deslocamento batocrômico do ligante Cis para o Trans com uma variação de aproximadamente 40 nm dos seus respectivos máximos de absorção e com um aumento no valor da absortividade molar da ordem de 10 vezes do ligante $\mathrm{Cis}$ para o Trans.

Em relação à atribuição de grupo cromofórico responsável pela transição, pode ser feito um paralelo com os dados disponíveis na literatura para o pseudo-oxocarbono croconato violeta. ${ }^{30,69-72}$ Estes estudos sugerem que o sistema eletrônico $\pi$ deslocalizado sobre o anel pentacíclico é o principal cromóforo responsável pela absorção mais intensa, indicando uma transição do tipo $\pi \rightarrow \pi^{*}$ a responsável pela banda $534 \mathrm{~nm}$; já a transição na região de maior energia em $445 \mathrm{~nm}$ seria atribuída pelos grupos dicianometileno. Assim, pode-se sugerir que a presença dos dois grupos dicianometileno na posição Cis exerça um novo tipo de interação molecular devido à proximidade e possíveis interações dos grupos substituintes. Essa mesma interação não ocorreria no caso do ligante Trans, uma vez que a distância dos mesmos não favorece uma interação efetiva significativa. A maior deslocalização eletrônica para a esquaraína Trans de sódio justificaria uma única transição eletrônica envolvendo os orbitais $\pi \rightarrow \pi^{*}$ centrada no anel e as demais seriam referentes a transições $\pi \rightarrow \pi^{*}$ do novo grupo cromofórico. A menor simetria em solução poderia explicar o fato de que no espectro eletrônico do composto Cis é observado um ombro próximo ao máximo de absorção atribuído à transição $\pi \rightarrow \pi^{*}$ do anel, sendo que esses orbitais estariam sofrendo uma interferência mais efetiva de orbitais dos grupos substituintes.

\section{CONCLUSÕES}

A aproximação feita pela análise de teoria de grupo às esquaraínas Cis e Trans substituídas se mostrou bem interessante para predizer o comportamento espectroscópico destas espécies, especialmente para o derivado Trans. A análise comparativa entre essas duas espécies é de grande relevância, pois aborda um tema extremamente teórico com aproximações fáceis de considerar e visualizar. A partir dos resultados percebe-se que uma abordagem mais ampla de discussão teve de ser considerada para verificação da simetria teórico/prática para a espécie Cis. Esse detalhe se mostrou interessante uma vez que propiciou uma discussão supramolecular e o impacto deste tipo de visão para explicar observáveis.

\section{MATERIAL SUPLEMENTAR}

Disponível em http://www.quimicanova.sbq.org.br, em formato PDF, com acesso livre.

\section{REFERÊNCIAS}

1. West, R.; Isr. J. Chem. 1980, 20, 300.

2. Seitz, G.; Imming, P.; Chem. Rev. 1992, 92, 1227.

3. de Oliveira, V. E.; Diniz, R.; de Oliveira, L. F. C.; Quim. Nova 2009, 32, 1917.

4. Zhao, B.; Back, M. H.; Can. J. Chem. 1991, 69, 528.

5. Andreola, S.; Gentile, G.; Battistini, A.; Cattaneo, C.; Zoja, R.; J. Forensic Sci. 2011, 56, 771.

6. Tian, J.; Cao, D.; Zhou, X.; Hu, J.; Huang, M.; Li, C.; ACS Nano 2018 , 12,3424

7. Liu, L.; Miao, L.; Li, L.; Li, F.; Lu, Y.; Shang, Z.; Chen, J.; J. Phys. Chem. Lett. 2018, 9, 3573.

8. Onaran, M. B.; Comeau, A. B.; Seto, C. T.; J. Org. Chem. 2005, 70, 10792.

9. Ramroop-Singh, N.; Narinesingh, D.; Singh, G.; Seto, C. T.; Comeau, A. B.; Bioorg. Chem. 2010, 38, 234.

10. Sherertz, E. F.; Sloan, K. B.; Arch. Dermatol. Res. 1988, $280,57$. 
11. Shinada, T.; Isida, T.; Ohfune, Y.; J. Synth. Org. Chem. Jpn. 2007, 65 30.

12. Tietze, L. F.; Arlt, M.; Beller, M.; Glüsenkamp, K.-H.; Jähde, E.; Rajewsky, M. F.; Chem. Ber. 1991, 124, 1215.

13. Tietze, L. F.; Arlt, M.; Beller, M.; Glüsenkamp, K. H.; Jahde, E.; Rajewsky, M. F.; Chem. Ber. 1991, 124, 1215.

14. C. Herndon, W.; Structure-resonance theory for oxocarbons, Vol. 103, 1983 , pp. 219.

15. Junqueira, G. M. A.; Rocha, W. R.; De Almeida, W. B.; Dos Santos, H F.; J. Mol. Struct.: THEOCHEM 2005, 719, 31.

16. Sakamoto, K.; Ihaya, Y. J.; J. Am. Chem. Soc. 1970, 92, 2636.

17. West, R. C.; Oxocarbons, Elsevier Science: Amsterdam, 2012.

18. Ito, M.; Takahashi, M.; Kaya, K.; Abstracts of Papers of the American Chemical Society 1979, 150

19. Ribeiro, M. C. C.; de Oliveira, L. F. C.; Santos, P. S.; Chem. Phys. 1997 $217,71$.

20. Takahashi, M.; Kaya, K.; Ito, M.; Chem. Phys. 1978, 35, 293.

21. Santos, P. S.; Amaral, J. H.; Deoliveira, L. F. C.; J. Mol. Struct. 1991 , $243,223$.

22. Lautié, A.; Lautié, M.-F.; Novak, A.; Can. J. Chem. 1985, 63, 1394.

23. Macintyre, W. M.; Werkema, M. S.; J. Chem. Phys. 1964, 40, 3563.

24. West, R.; Eggerding, D.; Perkins, J.; Handy, D.; Tuazon, E. C.; J. Am. Chem. Soc. 1979, 101, 1710.

25. Baenziger, N. C.; Hegenbarth, J. J.; J. Am. Chem. Soc. 1964, 86, 3250.

26. Cohen, S.; Cohen, S. G.; J. Am. Chem. Soc. 1966, 88, 1533.

27. West, R.; Niu, H.-Y.; Powell, D. L.; Evans, M. V.; J. Am. Chem. Soc. 1960, 82, 6204.

28. Artizzu, F.; Deplano, P.; Pilia, L.; Serpe, A.; Marchio, L.; Bernot, K.; Mercuri, M. L.; Inorg. Chim. Acta. 2011, 370, 474.

29. De Abreu, H. A.; Junior, A. L. S.; Leitao, A. A.; De Sa, L. R. V.; Ribeiro, M. C. C.; Diniz, R.; de Oliveira, L. F. C.; J. Phys. Chem. A 2009, 113, 6446.

30. Fatiadi, A. J.; J. Am. Chem. Soc. 1978, 100, 2586.

31. Goncalves, N. S.; Deoliveira, L. F. C.; Santos, P. S.; Spectrochim. Acta, Part A 1994, 50, 263.

32. Kurdiukova, I. V.; Kulinich, A. V.; Ishchenko, A. A.; New J. Chem. 2012 36, 1564.

33. Barczynski, P.; Dega-Szafran, Z.; Katrusiak, A.; Szafran, M.; J. Mol. Struct. 2012, 1018, 28.

34. de Oliveira, L. F. C.; Santos, P. S.; J. Mol. Struct. 1991, 245, 215.

35. de Oliveira, V. E.; de Carvalho, G. S.; Yoshida, M. I.; Donnici, C. L.; Speziali, N. L.; Diniz, R.; de Oliveira, L. F. C.; J. Mol. Struct. 2009, 936, 239.

36. de Oliveira, V. E.; Freitas, M. C. R.; Diniz, R.; Yoshida, M. I.; Speziall, N. L.; Edwards, H. G. M.; de Oliveira, L. F. C.; J. Mol. Struct. 2008, $881,57$.

37. Eggerding, D.; West, R.; J. Org. Chem. 1976, 41, 3904.

38. Galibert, A. M.; Soula, B.; Donnadieu, B.; Fabre, P. L.; Inorg. Chim. Acta 2001, 313, 160.

39. Liu, H.; Tomooka, C. S.; Moore, H. W.; Synth. Commun. 1997, 27, 2177.

40. Lopes, J. G. S.; de Oliveira, L. F. C.; Edwards, H. G. M.; Santos, P. S.; J. Raman Spectrosc. 2004, 35, 131.

41. Yeşilel, O. Z.; Odabaşoğlu, M.; Büyükgüngör, O.; J. Mol. Struct. 2008, $874,151$.

42. Eggerding, D.; West, R.; J. Am. Chem. Soc. 1975, 97, 207.

43. Eggerding, D.; West, R.; J. Am. Chem. Soc. 1976, 98, 3641.
44. Cowan, J. A.; Howard, J. A. K.; Acta Crystallogr., Sect. E 2004, 60, $\mathrm{m} 511$.

45. Lunelli, B.; Monari, M.; Bottoni, A.; J. Phys. Chem. A 2001, 105, 2257.

46. Sreejith, S.; Carol, P.; Chithra, P.; Ajayaghosh, A.; J. Mater. Chem. 2008, $18,264$.

47. Nyquist, R. A. In Interpreting Infrared, Raman, and Nuclear Magnetic Resonance Spectra, Academic Press, San Diego, 2001, p. 1.

48. Busetti, V.; Lunelli, B.; J. Phys. Chem. 1986, 90, 2052.

49. Pena, C.; Marie Galibert, A.; Soula, B.; Fabre, P.-L.; Bernardinelli, G.; Castan, P.; J. Chem. Soc., Dalton Trans. 1998, 239.

50. Law, K.-Y.; J. Phys. Chem. 1995, 99, 9818.

51. Corrêa, C. C.; Diniz, R.; Chagas, L. H.; Rodrigues, B. L.; Yoshida, M. I.; Teles, W. M.; Machado, F. C.; Edwards, H. G. M.; de Oliveira, L. F. C.; Vib. Spectrosc. 2007, 45, 82.

52. Silva, C. E.; Dos Santos, H. 1. F.; Speziali, N. L.; Diniz, R.; de Oliveira, L. F. C.; J. Phys. Chem. A 2010, 114, 10097.

53. Oswald, B.; Patsenker, L.; Duschl, J.; Szmacinski, H.; Wolfbeis, O. S.; Terpetschnig, E.; Bioconjugate Chem. 1999, 10, 925.

54. Interpreting Infrared, Raman, and Nuclear Magnetic Resonance Spectra, Nyquist, R. A., ed.; Academic Press, San Diego, 2001, pp. 1.

55. Bhagavantam, S.; Venkatarayudu, T. In Theory of Groups and its Application to Physical Problems; Bhagavantam, S., Venkatarayudu, T., eds.; Academic Press: San Diego, 1969, pp. 85.

56. Colthup, N. B.; Daly, L. H.; Wiberley, S. E., In Introduction to Infrared and Raman Spectroscopy (Third Edition); Colthup, N. B., Daly, L. H., Wiberley, S. E., eds.; Academic Press: San Diego, 1990, pp. 109.

57. Infrared and Raman Spectroscopy (Second Edition); Larkin, P. J., ed.; Elsevier: Amsterdam, 2018, pp. 7.

58. Infrared and Raman Spectroscopy (Second Edition); Larkin, P. J., ed.; Elsevier: Amsterdam, 2018, pp. 1.

59. Sala, O.; Fundamentos da espectroscopia Raman e no Infravermelho. Editora UNESP, 1996.

60. Neuse, E. W.; Green, B. R.; J. Org. Chem. 1974, 39, 3881.

61. Group Theory for Chemists (Second Edition); Molloy, K. C., ed.; Woodhead Publishing: Sawston, 2013,pp. 121.

62. Applied Group Theory; Cracknell, A. P., ed.; Pergamon: Oxford, 1968, pp. 3.

63. Symmetry in Bonding and Spectra; Douglas, B. E.; Hollingsworth, C. A., eds.; Academic Press: San Diego, 1985, pp. 1.

64. Symmetry and Group Theory in Chemistry; Ladd, M., ed.; Woodhead Publishing: Sawston, 1998, pp. 221.

65. Bonnett, R.; Motevalli, M.; Siu, J.; Tetrahedron 2004, 60, 8913.

66. de Oliveira, L. F. C. M., S. R.; Gonçalves, N. S.; Santos, P. S.; Quim. Nova 1992, 15, 55 .

67. Georgopoulos, S. L.; Diniz, R.; Yoshida, M. I.; Speziali, N. L.; Santos, H. F. D.; Junqueira, G. M. A.; de Oliveira, L. F. C.; J. Mol. Struct. 2006, $794,63$.

68. Griffiths, G. R.; Rowe, M. D.; Webb, G. A.; J. Mol. Struct. 1971, 8, 363.

69. Castro, L. F.; Almeida, T. C.; Soares, A. L.; Yoshida, M. I.; Machado, F. C.; Diniz, R.; de Oliveira, L. F. C.; Vib. Spectrosc. 2010, 54, 112.

70. Cappa de Oliveira, L. F.; da Silva Lopes, J. G.; Barone, P. M. V. B.; Ribeiro, M. C. C.; Santos, P. S.; J. Mol. Struct. 1999, 510, 97.

71. Makowski, M.; Pawlikowski, M. T.; Chem. Phys. Lett. 2003, 376, 631.

72. Smart, L. E.; Moore, E. A.; Solid state chemistry: an introduction, CRC Press: Boca Raton, 2005.

FAPESP contribui para custear a publicação deste artigo 